\title{
Getting ready to sit a HERMES examination: practical issues and tips
}

A HERMES examination in adult respiratory medicine or in paediatric respiratory medicine is a knowledge-based examination with 90 multiple-choice questions (MCQ) in English. Condidates can sit a HERMES examination during the ERS Annual Congress or locally in the Netherlands or Russia in collaboration with those countries' national societies.

\section{Why sit a HERMES examination?}

Each HERMES examination MCQ has a clinical focus reflecting real-life practice in adult or paediatric respiratory medicine. Taking a HERMES examination is a valuable benchmarking exercise for the participants. Each MCQ undergoes a rigorous process of formulation and validation by European representatives who ensure that state-of-the-art practices as well as authentic professional situations are illustrated. A participant is therefore given the opportunity to keep abreast of new developments in the field and verify and update their knowledge. If participating as a trainee, the examination can be used as a driver for learning. If sitting the examination for self-assessment, candidates can benchmark themselves against their peers and identify their potential educational needs. Participation in the examination in order to earn the European diploma demonstrates commitment to life-long learning and continuous professional development. Several positive actions follow the decision to take a HERMES examination: 1) getting ready by studying for the examination; 2) sitting the examination itself; 3) identifying potential educational needs based on the results; and 4) planning and attending educational activities to address these needs. These positive actions are geared towards ensuring the best possible care for patients, which is the ultimate goal of all HERMES programmes.

\section{How is a HERMES examination paper produced?}

Producing an actual HERMES examination paper follows a rigorous process with strict quality control in collaboration with the Institute of Medical Education at the University of Berne in Switzerland, the ERS examination committees and the MCQ authors. Everyone involved in the production of the HERMES examination paper is committed to ensuring the quality of the questions as well as the scientific merits of each step in the processes.

Every year, an MCQ writing author workshop takes place at the ERS Annual Congress where interested respiratory medicine experts are invited to learn how to formulate MCQs following the HERMES examination formats. The participants then submit MCQs which undergo formal review, content review and finally revision in a face-to-face workshop with
Statement of Interest $J$-L. Noël is an employee of ERS 


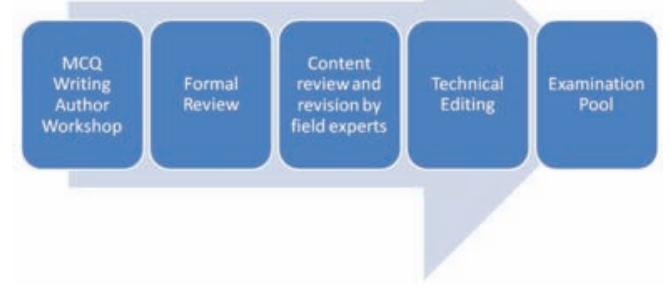

Figure 1

MCQ production steps. From this examination pool, the examination committee selects go questions which will be the year's question set and printed in the examination booklet.

field experts. Upon revision, technical editing is done on each question before they go into the examination pool in their final form (figures. 1 and 2).

\section{What are the HERMES MCQ formats?}

MCQs in a HERMES examination follow two formats: 1 ) type $A$; and 2) type K prime. Type $A$ questions require only one best answer out

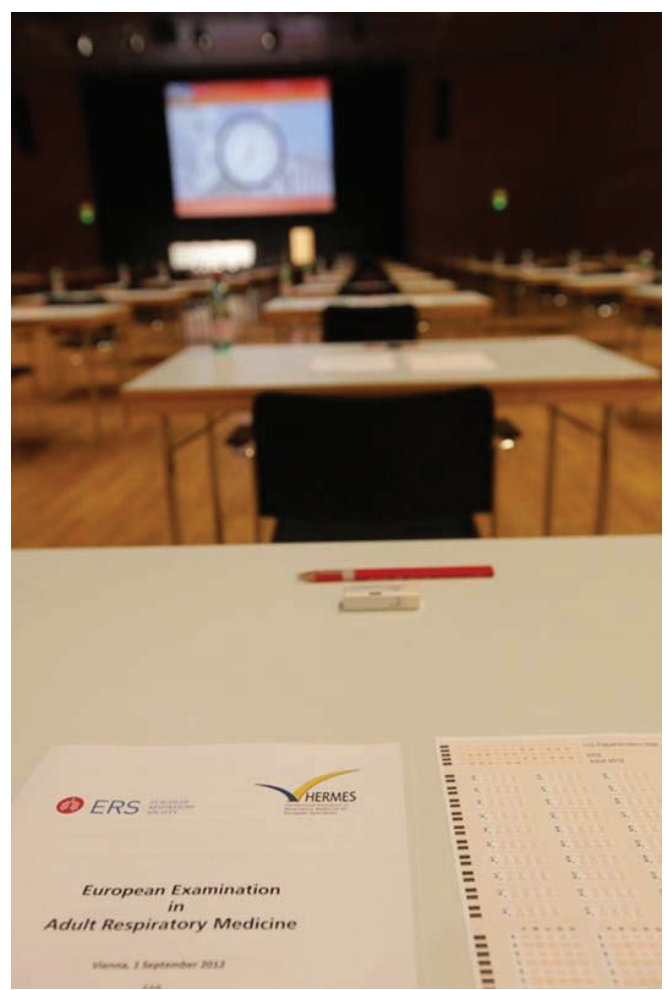

Figure 2

Examination booklet. of five options whereas type $\mathrm{K}$ prime format MCQs require multiple correct or incorrect decisions. To each question or incomplete statement, there are four answers or statement completions. A decision for each of the four options is required whether they are correct or incorrect and is marked with a "+" or "-" on the answer sheet.

The box opposite illustrates are actual items in the two formats. These and others can be accessed from the online sample of the HERMES examination in Paediatric Respiratory Medicine (www.ers-education.org/MOS_R7/ MosPub/HERMES_Test/index.html).

\section{What is the examination blueprint?}

HERMES examinations are designed and constructed using their respective examination blueprints.

These blueprints identify the topics covered in each examination and are arranged in 2 dimensions: 1) diseases and 2) medical actions. These are based on the European syllabi [1, 2]. The blueprints also provide information on the proportion of questions in the examination covering each topic.

\section{Which existing resources can help in preparing for the examination?}

\section{Guidelines}

HERMES examinations strive to illustrate stateof-the-art and current best practices in respiratory medicine. Current guidelines are therefore the basis of the questions in patient management, whether in differential diagnosis, diagnostic procedures, therapy, etc. The ERS guidelines are collected at www.ers-education. org (fig. 3).

\section{ERS Handbook of Respiratory Medicine}

This is a concise, compact and easy-to-read guide to each of the key areas in respiratory medicine. Its 17 chapters are written by clinicians and researchers, providing a reference that covers everything from the basics to the latest developments in respiratory medicine. Handbook users also have access to online CME questions. This reference is a key resource 


\section{HERMES MCQ formats}

\section{Type A}

A 2-year-old boy is presented with a 3-month history of pallor and decreasing growth. He has had three admissions to the hospital, two with a lower respiratory tract infection and one with diarrhoea. His appetite was good and his bowel frequency was normal although his parents had noted his stools to be pale and greasy from time to time. On examination, his height was on the 0.4 th centile and weight below the 0.4 th centile, he had bottle caries, scanty crackles were heard in the right lower lobe, heart sounds were normal, abdomen soft and early finger clubbing was noted.

Investigations:

Blood

Haemoglobin $10.5 \mathrm{~g} \cdot \mathrm{dL}^{-1}$

White cell count $4.0 \times 10^{9} \cdot \mathrm{L}^{-1}$

Neutrophils $0.5 \times 10^{9} \cdot \mathrm{L}^{-1}$

Lymphocytes $3.5 \times 10^{9} \cdot \mathrm{L}^{-1}$

Eosinophils $0.5 \times 10^{9} \cdot \mathrm{L}^{-1}$

Platelets $110 \times 10^{9} \cdot \mathrm{L}^{-1}$

IgA, IgM, IgG, IgE normal

Pancreatic elastase $120 \mu \mathrm{g} \cdot \mathrm{L}^{-1}$

IgA anti-tissue transglutaminase antibodies negative Which of the following is the most likely diagnosis?

(A) Coeliac disease

(B) HIVIAIDS

(C) Intolerance to cow's milk

(D) Shwachman-Diamond syndrome

(E) X-linked agammaglobulinaemia

ANSWER: D

\section{Type K prime}

A previously healthy 3-month-old boy three days ago developed a runny nose, mild cough, low-grade fever and noisy breathing, without feeding. On examination, he showed a respiratory rate of 40 breaths per minute, stridor, and retractions. Flexible endoscopy showed the picture which is indicative of a subglottic haemangioma.

Which of the following options is/are appropriate at this stage?

(A) Oral propranolol

(B) Systemic corticosteroids

(C) Intra-lesional cidofovir

(D) Endoscopic laser resection

ANSWER: YES/YES/NO/YES for the examination committee in formulating questions. The ERS Handbook of Respiratory Medicine is a helpful resource and an important tool for those preparing to take the examination in adult respiratory medicine (fig. 4).

\section{Self-Assessment in Respiratory Medicine}

Seft-Assessment in Respiratory Medicine contains 111 MCQs covering the full-breadth of the specialty using clinical vignettes. The MCQs have been compiled and tested by the ERS Adult HERMES examination committee. Each MCQ was produced according to the rigorous process of production in the HERMES examination making Self-Assessment in Respiratory Medicine a perfect revision aid for HERMES examination participants (fig. 4).

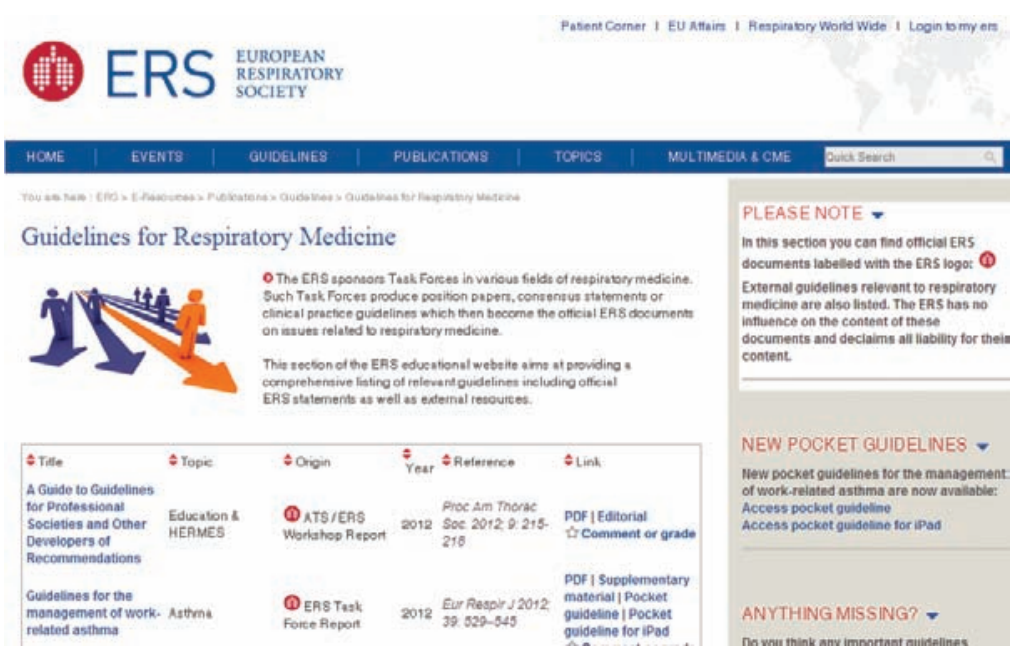

Figure 3

ERS guidelines documents online. 
(iii) ERS/handbook

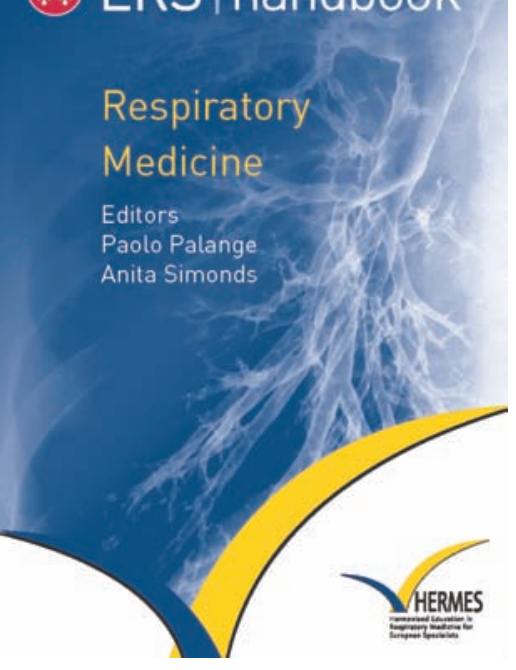

\section{fiti ERS/ handbook}

Self-Assessment

in Respiratory

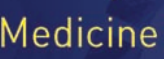

Editors

Konrad E. Bloch

with Paolo Palange and

Anita K. Simonds

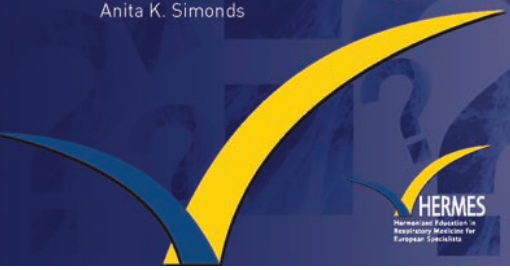

\section{Figure 4}

The ERS Handbook of Respiratory Medicine and the ERS Handbook of Self-Assessment in Respiratory Medicine.

\section{Paediatric examination online questions}

The ERS Paediatric HERMES Examination Committee compiled and tested a total of 30 MCQs online. The online test is designed to simulate the actual HERMES examination in Paediatric Respiratory Medicine and the 30 questions have undergone the same stringent quality control as all the MCQs in the examination. To replicate examination conditions, the online test should be completed within one hour. The aim is to give insight into

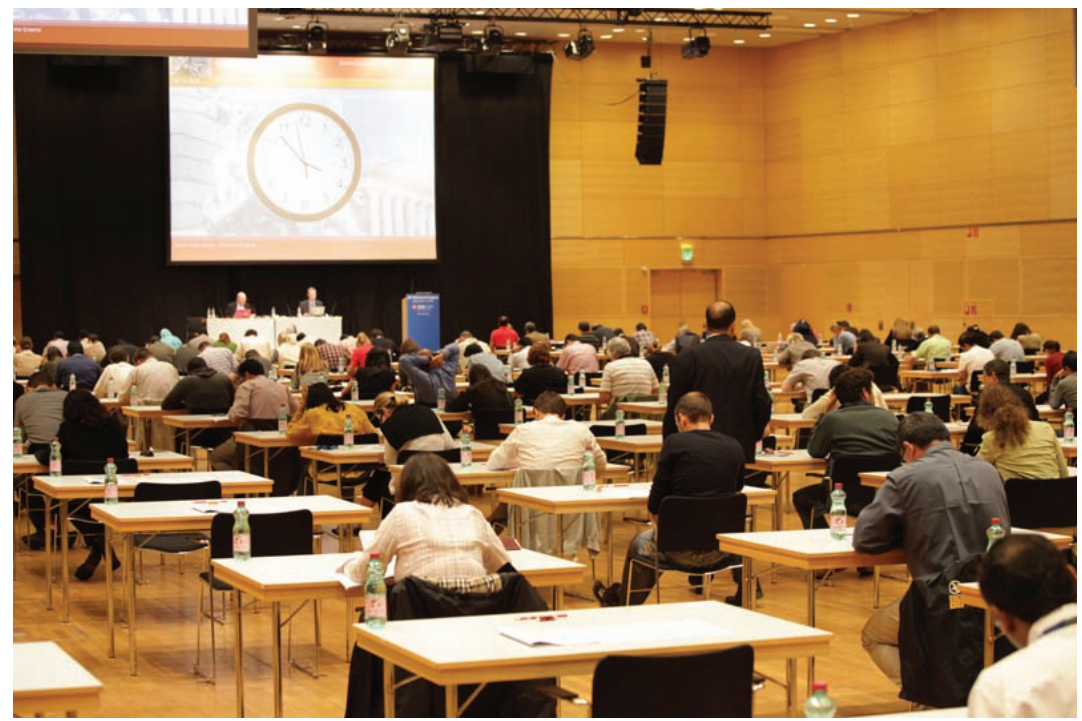

Figure 5

2012 HERMES examinations. the format, difficulty level and a sample of what is being asked in each MCQ. Visit hermes. ersnet.org/examinations/paediatric-examinationpreparation-and-mentorship-2.html

\section{HERMES Diplomates Mentorship}

In order to help examination candidates in their preparation and revision, the ERS has launched HERMES diplomates mentorship. Registered candidates will have the opportunity to interact with HERMES diplomates who have successfully passed HERMES examinations. HERMES diplomates share their experiences of taking the HERMES examination, including giving tips in good preparation. Visit hermes.ersnet.org for more details.

\section{Why attend the ERS HERMES Summer School or ERS endorsed courses?}

The ERS HERMES Summer School on 12-16 June 2013 in Barcelona intends to provide a comprehensive overview of each of the major fields of respiratory medicine based on the HERMES syllabus. This 5-day interactive course led by a world-class faculty will help participants prepare for the HERMES examination in adult respiratory medicine. The programme is designed to challenge participants and provide a learning framework, including interactive sessions and MCQ discussion sessions. The course is delivered through a range of media including lectures, workshops and case-based discussions. Sessions will be dedicated to discussion and answering MCQs, which have been prepared by the HERMES examination committee. Educational materials are distributed on site, including the ERS Handbooks.

National societies can deliver state-of-the-art training programmes to help prepare candidates to sit the HERMES European examination in adult respiratory medicine. The ERS provides guidelines to creating a structured educational programme listing the core components of the HERMES syllabus in adult respiratory medicine. Teaching methods and other essential specifications required to deliver a high-quality educational programme are included in the guidelines. The ERS endorses these educational programmes. In 2012, courses were organised by the Italian Society of Respiratory Medicine 
(SIMeR) and the Hellenic Thoracic Society Details of future ERS endorsed courses will be available at hermes.ersnet.org.

\section{I3 HERMES examinations}

Registrations for the 2013 HERMES examinations in Adult Respiratory Medicine and
Paediatric Respiratory Medicine are open (fig. 5). The registration deadline is 30 June 2013. For the local HERMES examinations, contact the Dutch Thoracic Society at www. nvalt.nl for information on the in-training assessment in Adult Respiratory Medicine in the Netherlands and the Russian Respiratory Society at www.pulmonology.ru for in-training assessment and self-assessment in Russia.

\section{Reference}

1. Loddenkemper R, Séverin T, Eiselé J-L, et al. HERMES: a European core syllabus in respiratory medicine. Breathe 2006; 3: 59-70.

2. Gappa M, Noël J-L, Severin T, et al. Paediatric HERMES: a European syllabus in paediatric respiratory medicine. Breathe 2009; 5: 237-241. 